سلوك العتبات المركبة (حديد-خرسانة) المقواة بشرائط (CFRP) تحت تأثير الأحمال التكرارية المفئة

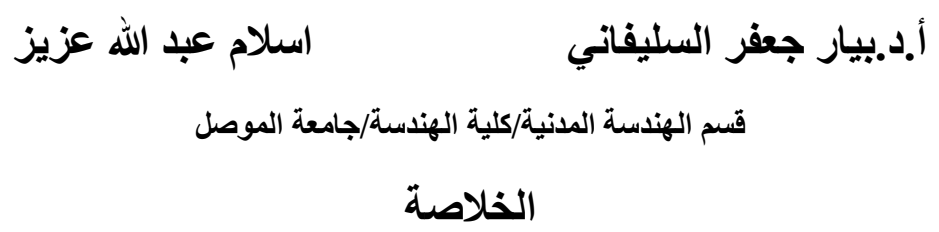

يثمل البحث الحالي دراسة عملية تهدف الى التعرف على سلوك الأعتاب المركبة المقواة بشرائط البوليمر المسلحة

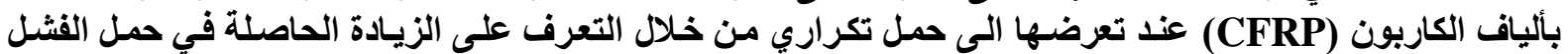

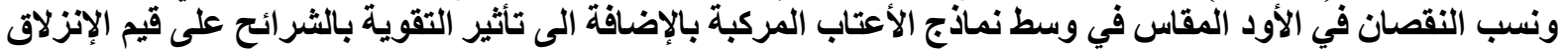

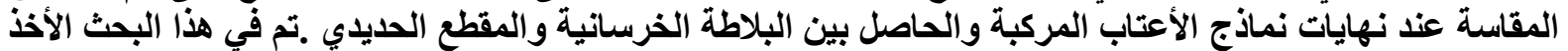

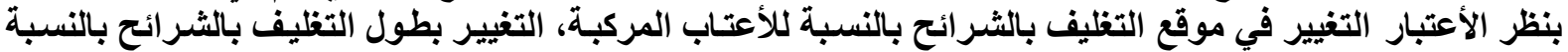

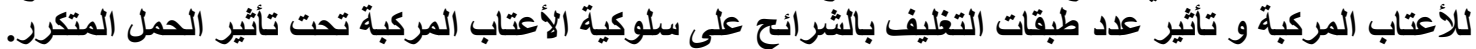

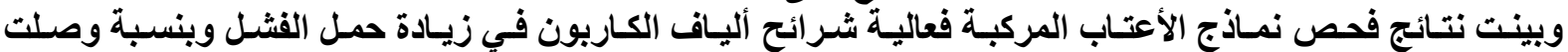

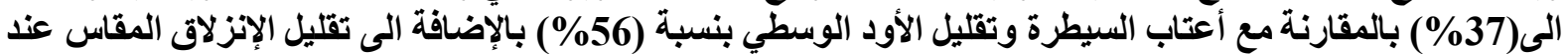

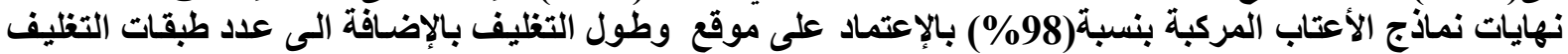
بشرائح ألياف الكاربون.

الكلمات الدالة:الأعتاب المركبة،ألياف الكاربون،الحمل التكراري،الإنزلاق.

\title{
Behavior Of Composite Beams (Steel-Concrete) Strengthened By (CFRP) Sheets Under Repeated Loading
}

\author{
Dr. Bayar J. Al-Sulayfani
}

Islam Abd-Ullah Aziz

Civil Engineering Dept./ College of Engineering/ University of Mosul

\begin{abstract}
Present study aims mainly to conduct the behavior of composite beams strengthened with carbon fiber reinforced polymers (CFRP) as they subjected to repeated loads. It investigates the enhancement of load capacity up to failure and the reduction in transverse deflection at the ends of composite beam as affected by (CFRP) strengthening. The following parameters were taken in to consideration in this paper. Location of (CFRP) along the composite beam wrapped length effect and effects the Number of (CFRP) layers.

From test results it is obvious that (CFRP) is very active in increasing the load capacity by $(36 \%)$ and reducing the mid span deflection by $(56 \%)$ in addition to minimizing the slip at beam ends by $(98 \%)$. Depending on the location, length and number of (CFRP) layers in to account.
\end{abstract}

Key words:- Composite beams, CFRP, Repeated loads and Slip. 


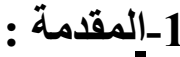

إن للمنشأ المركب ميزات جعلتة بأخذ حيز اً كبيرًا في مجال البناء وخاصةً في الأبنية المتعددة الطو ابق و الجسور

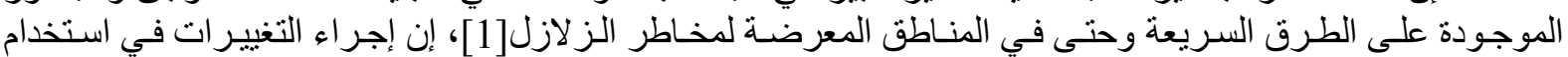

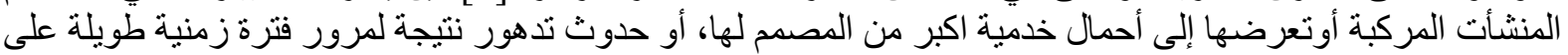

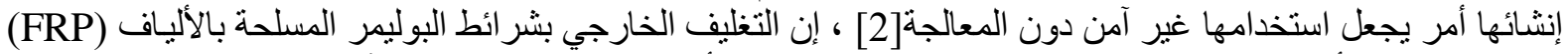

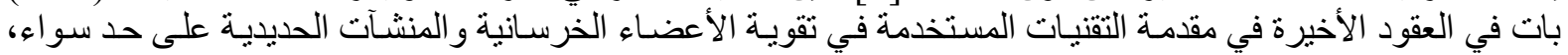

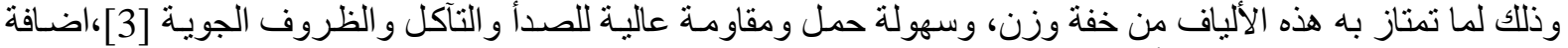

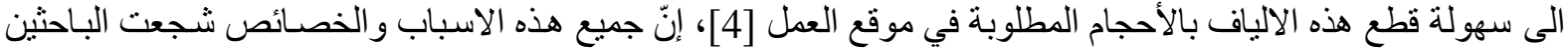

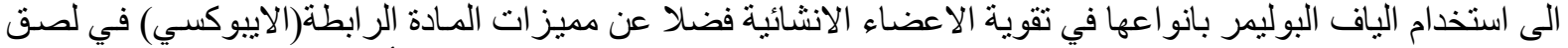

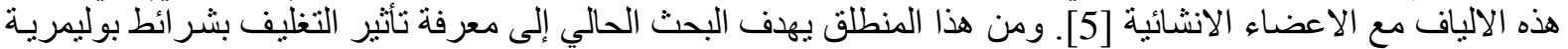

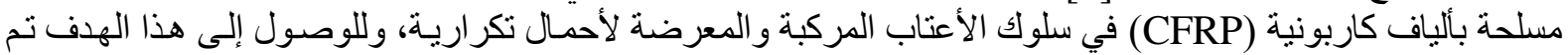

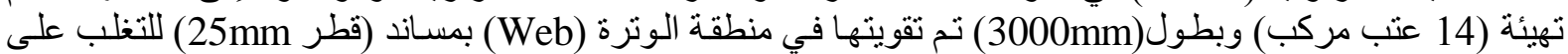

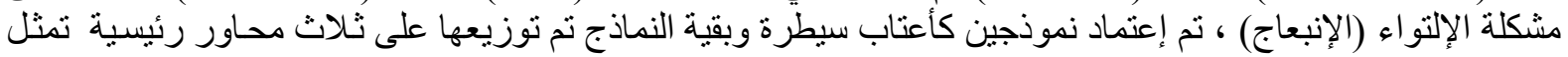

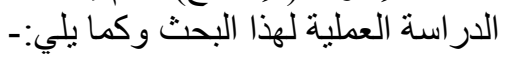

1- المحور الأول:التعرف على موقع التغليف لنمـاذج الأعتاب المركبة بشر ائط (CFRP) لإعطاء أفضـل النتائج لمقاومـة

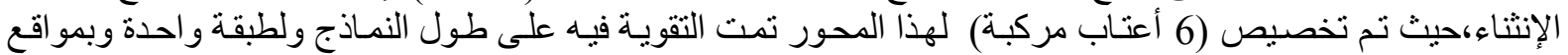
مختلفة. 2- المحور الثاني: التعرف على الطول الأمثل للتغليف بشر ائط (CFRP) ، وخصص لهذا الجزء(3 أعتاب مركبة) تم التغليف فيها بأطو ال مختلفة.

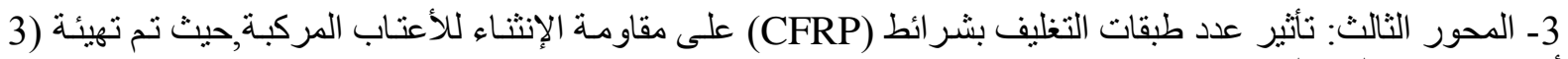

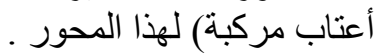

ولسهولة التعرف على العتبات المركبة المقو اه بشر ائط ألياف الكاربون رُمز الى هذه العتبات بالصيغة الموضحة في الثكل

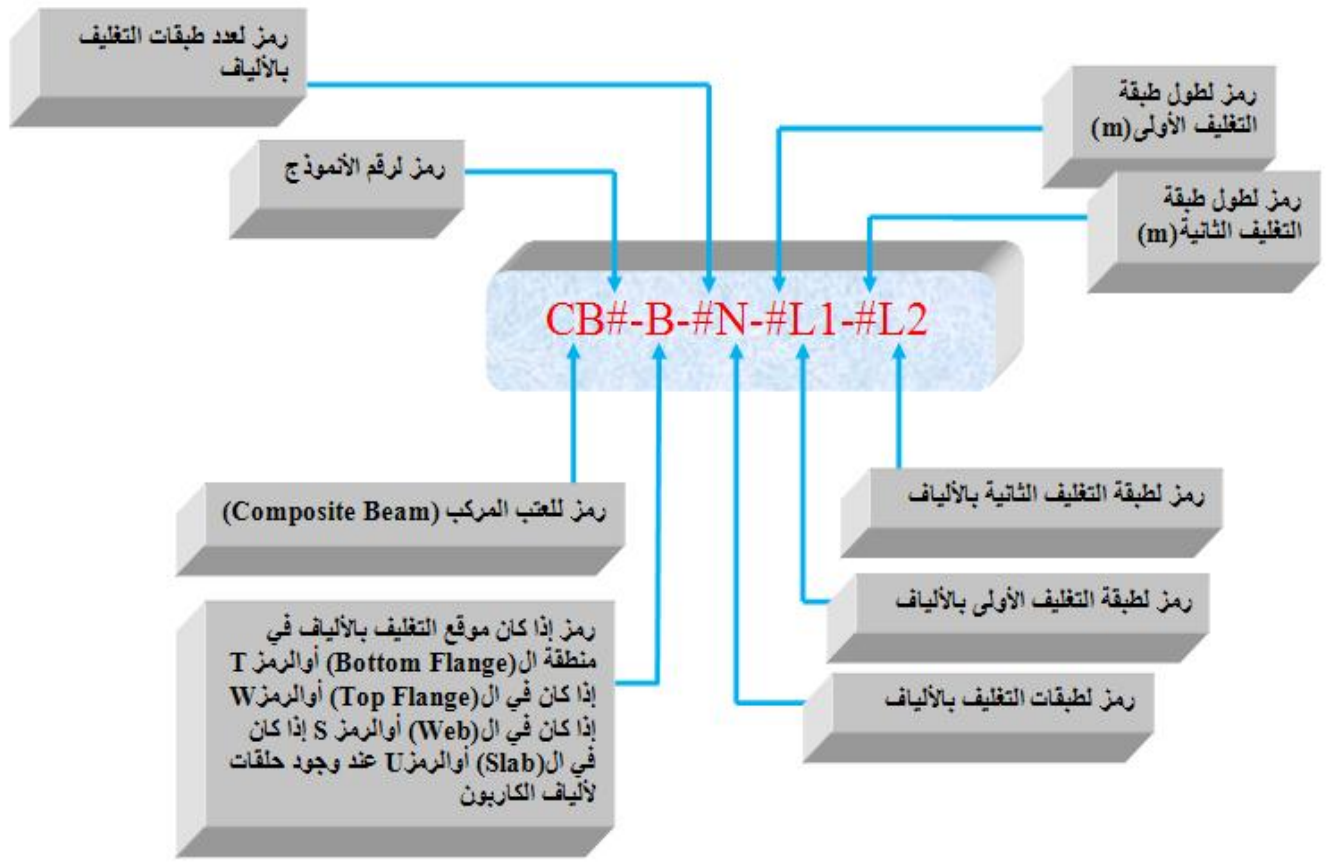

شكل(1): يوضح تفاصيل ترميز نماذج الأعتاب المركبة. 
ألسليفاني: سلوك العتبات المركبة (حديدخرسانة) المقواة بثرائط (CFRP) تحت تأثير الأحمال التكرارية

و الجدول التالي يوضح تفاصيل عدد ورموز ومناطق التقوية لمجاميع نماذج الأعتاب المركبة المستخدمة في هذا البحث جدول(1): مجاميع وعدد ورموز ومواقع التقوية للأعتاب المركبة المقواه بشرائط (CFRP).

\begin{tabular}{|c|c|c|c|}
\hline Groups & No.of Composite Beams & Specimen designation & Strengthened Region \\
\hline \multirow{2}{*}{ A } & \multirow{2}{*}{2} & CCB1 & \multirow{2}{*}{ Reference } \\
\hline & & CCB2 & \\
\hline \multirow{6}{*}{ B } & \multirow{6}{*}{6} & CB1-B-1N-3L1 & Bottom Flange \\
\hline & & CB2-B-1N-3L1 & Bottom Flange \\
\hline & & CB3-BW-1N-3L1 & Bottom Flange\&Web \\
\hline & & CB4-BU-1N-3L1 & Bottom Flange\&Web \\
\hline & & CB5-TS-1N-3L1 & Top Flange\&Slab \\
\hline & & CB6-BTS-1N-3L1 & Top Flange\&Slab\& Bottom Flange \\
\hline \multirow{3}{*}{$\mathbf{C}$} & \multirow{3}{*}{3} & CB7-BTS-1N-2L1 & \multirow{3}{*}{ Top Flange\&Slab\& Bottom Flange } \\
\hline & & CB8-BTS-1N-1L1 & \\
\hline & & CB9-BTS-1N-1.5L1 & \\
\hline \multirow{3}{*}{ D } & \multirow{3}{*}{3} & CB10-BTS-2N-3L1-3L2 & \multirow{3}{*}{ Top Flange\&Slab\& Bottom Flange } \\
\hline & & CB11-BTS-2N-3L1-2L2 & \\
\hline & & CB12-BTS-2N-2L1-2L2 & \\
\hline
\end{tabular}

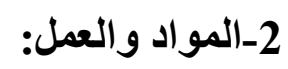
1-2:الاسمنت:

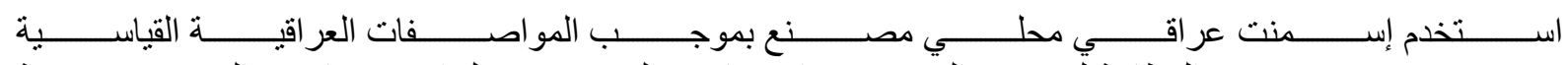
[6] المطابقة للإسمنت البورناندي الاعتيادي و المنتج في معمل إسمنت بادوش/التوسيع في مدينـة

2-2:2 الركام الناعم (الرمل) والركام الخشن (الحصى):

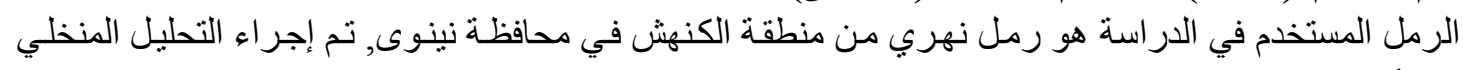

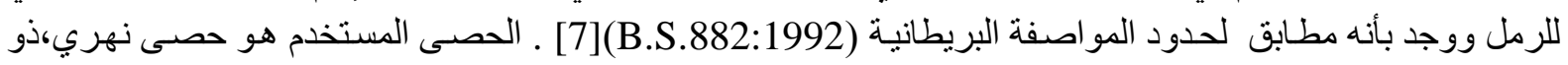

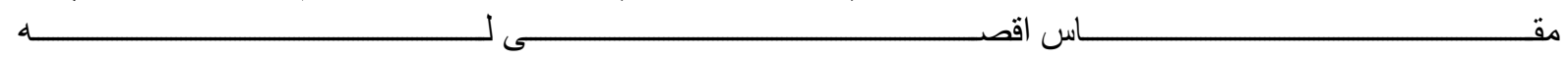
(20mm) (Max.agg size) (5-20mm) [ للركام الخشن المدرج من فن (B.S.882:1992)

3-2

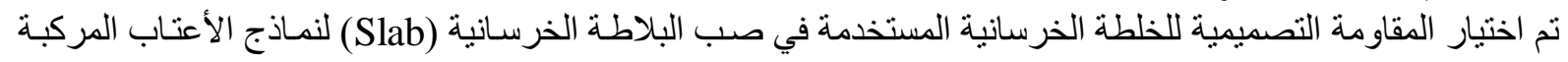

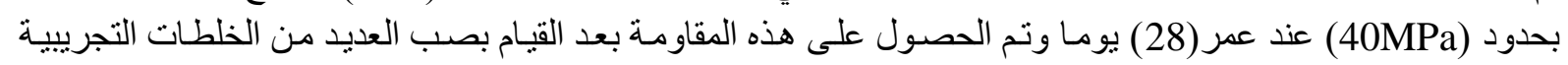

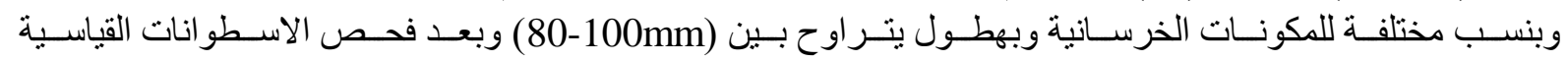

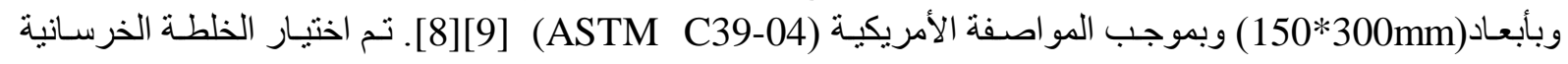
وبنسب وزنية(1:2.7:2.9/0.51).

4-2

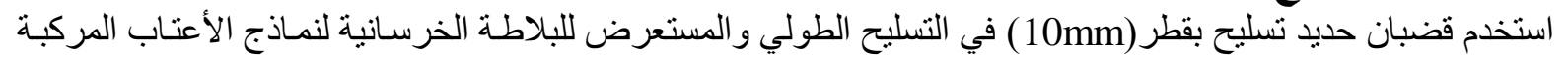

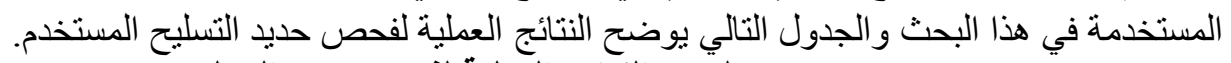
جدول(2):النتائج العملية لفحص حديد التسليح

\begin{tabular}{|c|c|c|c|c|c|}
\hline $\begin{array}{c}\text { Steel } \\
\text { Specimen } \\
(\mathbf{m m})\end{array}$ & $\begin{array}{c}\text { Yield } \\
\text { Strength(MPa) }\end{array}$ & $\begin{array}{c}\text { Yield } \\
\text { Strain }\end{array}$ & $\begin{array}{c}\text { Ultimate } \\
\text { Strength(MPa) }\end{array}$ & $\begin{array}{c}\text { Ultimate } \\
\text { Strain }\end{array}$ & $\begin{array}{c}\text { Modulus of } \\
\text { Elasticity(MPa) }\end{array}$ \\
\hline $\mathbf{1 0}$ & $\mathbf{5 0 5}$ & $\mathbf{0 . 0 0 2 6}$ & $\mathbf{6 1 0}$ & $\mathbf{0 . 0 1 2 9}$ & $\mathbf{1 9 4 2 3 0}$ \\
\hline
\end{tabular}




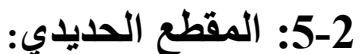

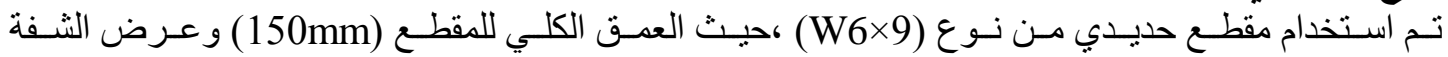

(100mm) وبطول (3000mm) و الجدول النالي(3) يوضح الخصائص الهندسية للمقطع الحديدي المستخدم و الجدول(4) يوضح نتائج الفحص العملية لثر ائح مأخوذة من المقطع الحديدي .

جدول(3):الخصائص الهندسية للمقطع الحديدي المستخدم:[10].

\begin{tabular}{|c|c|c|c|c|c|c|c|c|}
\hline \multirow{2}{*}{ Designation } & $\begin{array}{c}\text { Area } \\
\left(\mathbf{m m}^{2}\right)\end{array}$ & $\begin{array}{c}\text { Depth } \\
(\mathbf{m m})\end{array}$ & $\begin{array}{c}\text { Thickness } \\
\mathbf{t}_{\mathbf{w}} \\
(\mathbf{m m})\end{array}$ & $\begin{array}{c}\text { Width } \\
\mathbf{b}_{\mathbf{f}} \\
(\mathbf{m m})\end{array}$ & $\begin{array}{c}\text { Thickness } \\
\mathbf{t}_{\mathbf{f}} \\
(\mathbf{m m})\end{array}$ & $\begin{array}{c}\text { Nominal } \\
\text { weight } \\
\mathbf{p e r}(\mathbf{m}) \\
(\mathbf{K g})\end{array}$ & $\begin{array}{c}\mathbf{I}_{\mathbf{x}} \\
\left(\mathbf{m m}^{4}\right)\end{array}$ & $\begin{array}{c}\mathbf{I}_{\mathbf{y}} \\
\left(\mathbf{m m}^{4}\right) \\
\times \mathbf{1 0}^{6}\end{array}$ \\
\hline $\mathbf{W 6} \times \mathbf{9}$ & $\mathbf{1 6 9 7}$ & $\mathbf{1 5 0}$ & $\mathbf{4 . 5}$ & $\mathbf{1 0 0}$ & $\mathbf{5 . 5}$ & $\mathbf{1 3 . 5}$ & $\mathbf{6 . 8 2 6}$ & $\mathbf{0 . 9 1 1}$ \\
\hline
\end{tabular}

\begin{tabular}{|c|c|c|c|c|c|}
\multicolumn{7}{|c|}{ (النتائج العملية لفحص المقطع الحديدي. } \\
\begin{tabular}{|c|c|c|c|} 
Steel \\
Specimen
\end{tabular} & $\begin{array}{c}\text { Yield } \\
\text { Strength(MPa) }\end{array}$ & $\begin{array}{c}\text { Yield } \\
\text { Strain }\end{array}$ & $\begin{array}{c}\text { Ultimate } \\
\text { Strength(MPa) }\end{array}$ & $\begin{array}{c}\text { Ultimate } \\
\text { Strain }\end{array}$ & $\begin{array}{c}\text { Modulus of } \\
\text { Elasticity(MPa) }\end{array}$ \\
\hline W6×9 & 320 & $\mathbf{0 . 0 0 1 5 6}$ & 355 & 0.0155 & 204000 \\
\hline
\end{tabular}

6-2

تم إستخدام نوعين من المأدة الرابطة (الإيبوكسي) في هذا البحث لكون شر ائط البوليمر المستخدمة تم لصقها

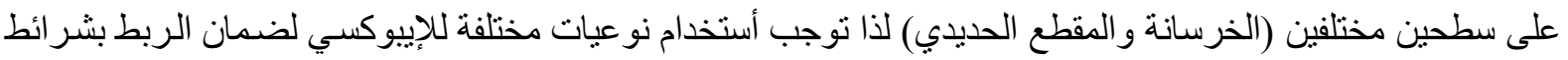
البوليمر و الأنواع هي (Sikadur 30) و (Sikadur 330) ـ

*): (المادة الرابطة (الإيبوكسي) (Sikadur 330):

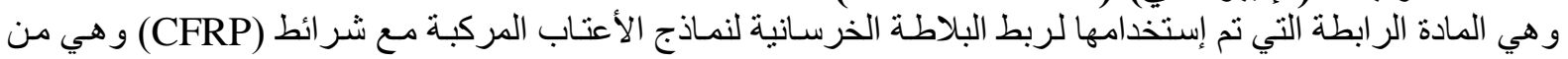

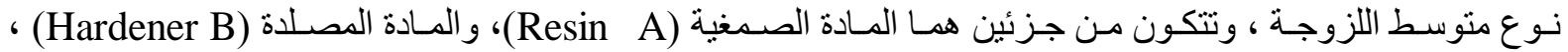

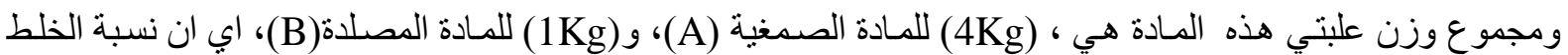

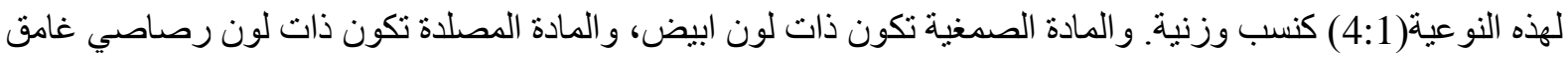

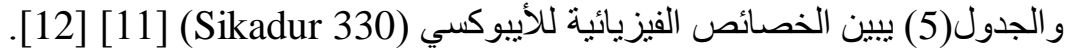

الجدول(5): يُبين الخصائص الفيزيائية للايبوكسي(Sikadur 330).

\begin{tabular}{|c|c|}
\hline DENSITY $\left(20^{\circ} \mathrm{C}\right)$ & $1.31 \mathrm{KG} / \mathrm{L}(\mathrm{COMP} . \mathrm{A}+\mathrm{B})$ \\
\hline Pot life & $+30 \mathrm{C}: 35 \mathrm{~min} . /+10 \mathrm{C}: 90 \mathrm{~min}$. \\
\hline Tensile strength & Curing 7 day, $+23^{\circ} \mathrm{C} / 30 \mathrm{~N} / \mathrm{mm}^{2}$ \\
\hline Flexural-E- Modulus & Curing 7 day, $+23^{\circ} \mathrm{C} / 3800 \mathrm{~N} / \mathrm{mm}^{2}$ \\
\hline
\end{tabular}

* (المادة الرابطة (الإيبوكسي) (Sikadur 30):

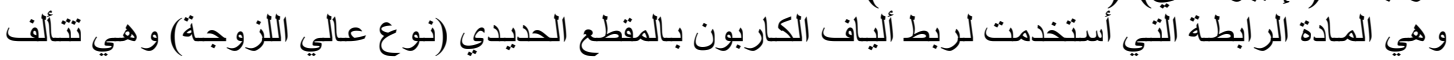

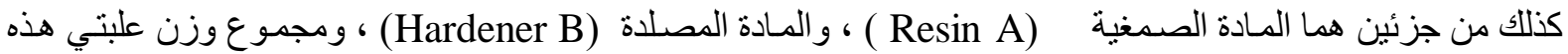

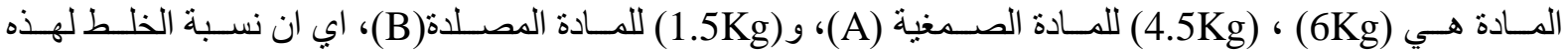

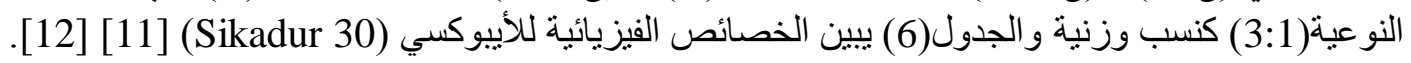

الجدول(6): يُيبين الخصائص الفيزيائية للايبوكسى(Sikadur 30).

\begin{tabular}{|c|c|c|c|c|}
\hline Sikadur type & Density & $\begin{array}{c}\text { Modulus of } \\
\text { elasticity }\end{array}$ & $\begin{array}{c}\text { Tensile bending } \\
\text { strength }\end{array}$ & Shear strength \\
\hline Sikadur-30 & $1.77 \mathrm{~kg} / \mathrm{l}$ & $12800 \mathrm{MPa}$ & $4 \mathrm{MPa}$ & $15 \mathrm{MPa}$ \\
\hline
\end{tabular}




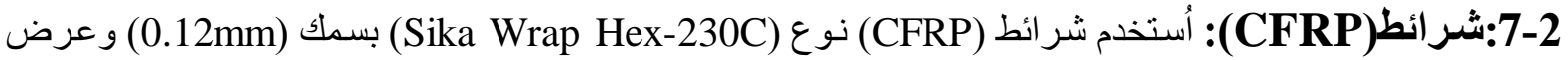
(300mm) و الجدول (7). يوضح الخصائص الفيزيائية للثر ائط المستخدمة [11] [12]. جدول(7):الخصائص الفيزيائية لثرائط (CFRP).

\begin{tabular}{|c|c|}
\hline FIBER TYPE & HIGH STRENGTH CARBON FIBERS \\
\hline A real weight & $220 \mathrm{gm} / \mathrm{m}^{2} \pm 10 \mathrm{gm} / \mathrm{m}^{2}$ \\
\hline Fiber density & $1.78 \mathrm{gm} / \mathrm{cm}^{3}$ \\
\hline Fabric design thickness & $0.12 \mathrm{~mm}($ Based on total carbon content $)$ \\
\hline Tensile strength of fibers & $4,100 \mathrm{~N} / \mathrm{mm}^{2}($ nominal$)$ \\
\hline Tensile E- modulus of fibers & $231,000 \mathrm{~N} / \mathrm{mm}^{2}($ nominal $)$ \\
\hline Strain at break of fibers & $1.7 \%($ nominal $)$ \\
\hline
\end{tabular}

3- تهيئة النماذج وآلية الفحص:-

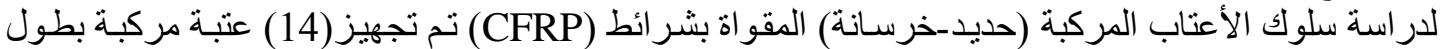

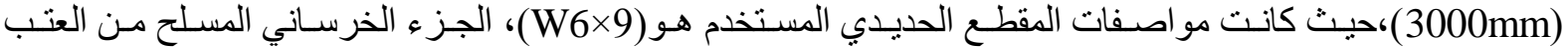

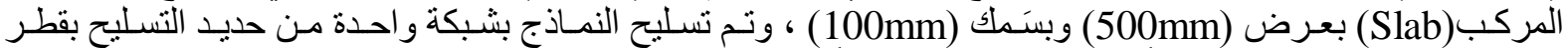

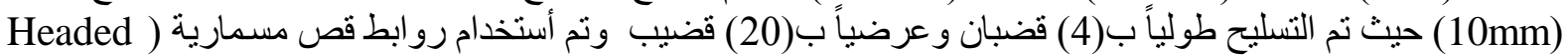
بقطر (12.5mm) و إرتفاع (50mm) وتم توزيعها على صفين ولحمها على الجزء العلوي من المقطع الحديدي

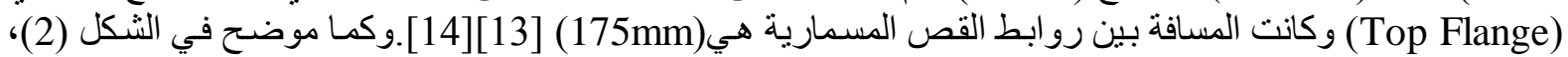

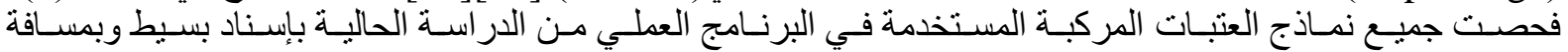
(2800mm) تم تثبيت جهاز (Deflection) النموذج وجهاز أخر عند أحد نهايـات النموذج لقياس الإنزلاق (Slip) بين البلاطـة الخرسانية والمقطع الحديدي بالإتجاة

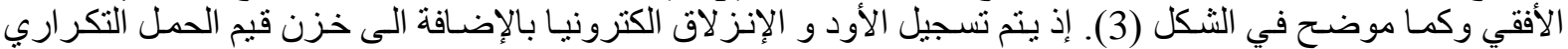

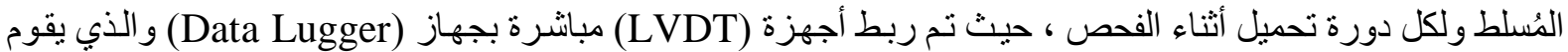

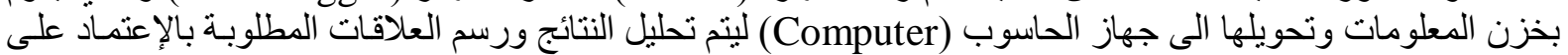

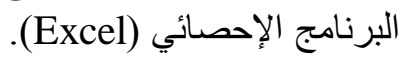

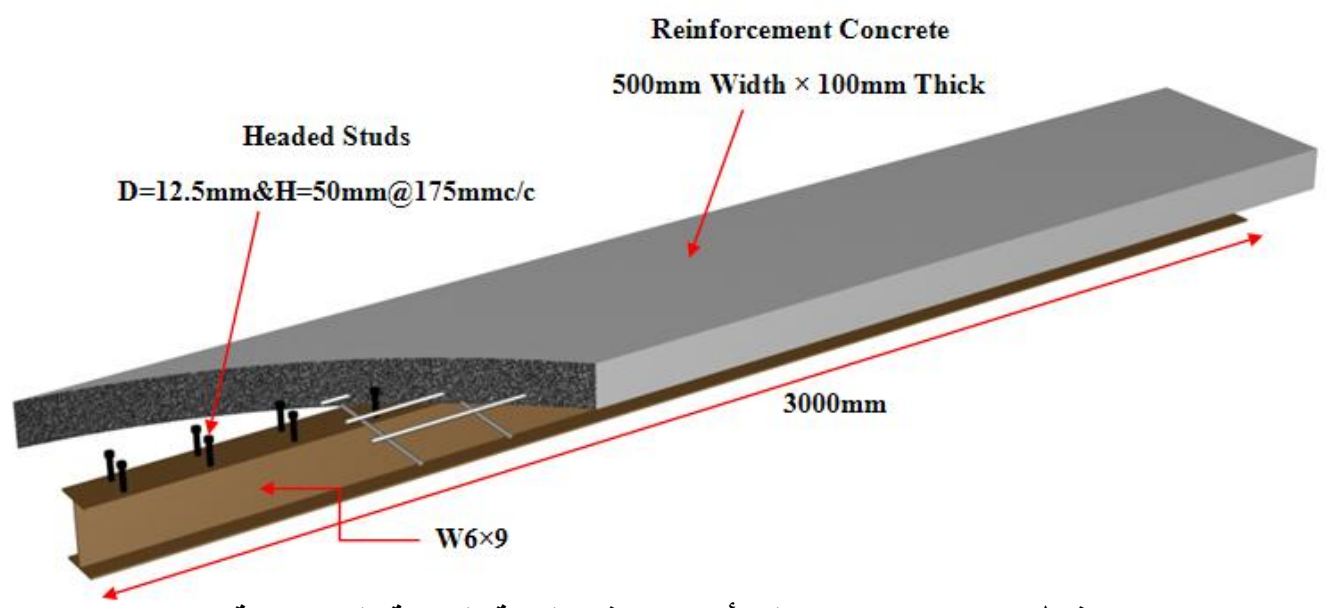

شكل(2): يوضح تفاصيل وأبعاد نموذج العتبة المركبة المستخدمة. 


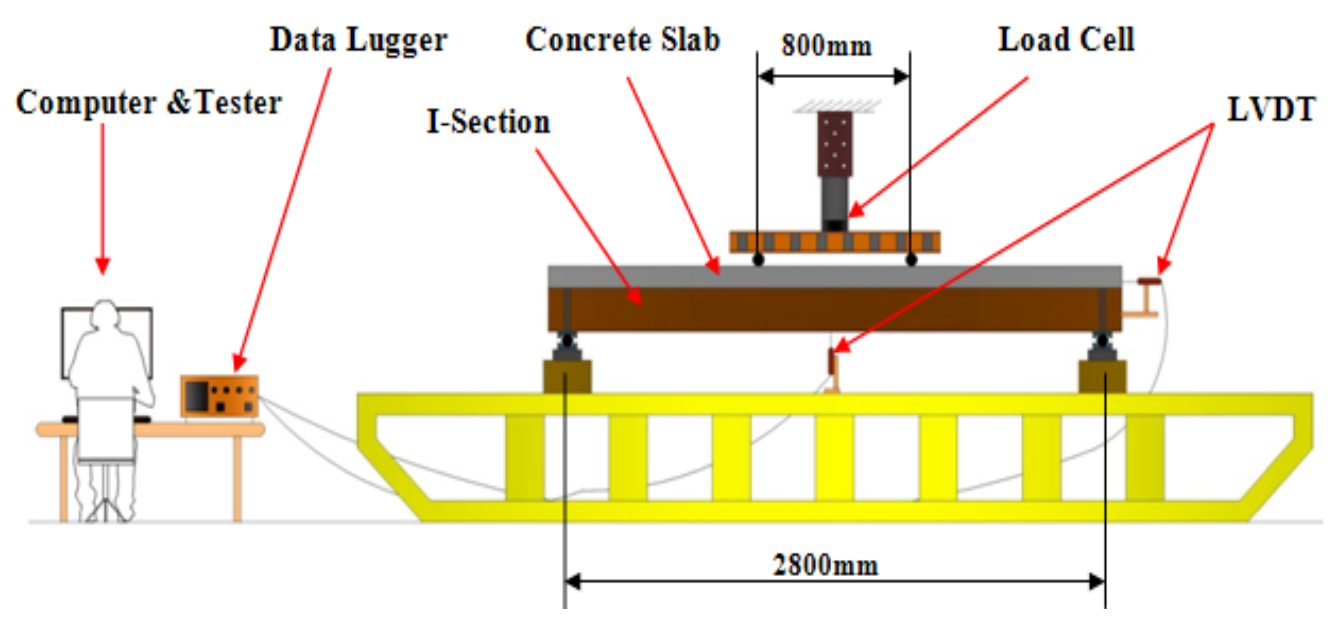

شكل(3): يوضح تفاصيل فحص نماذج العتبات المركبة المستخدمة.

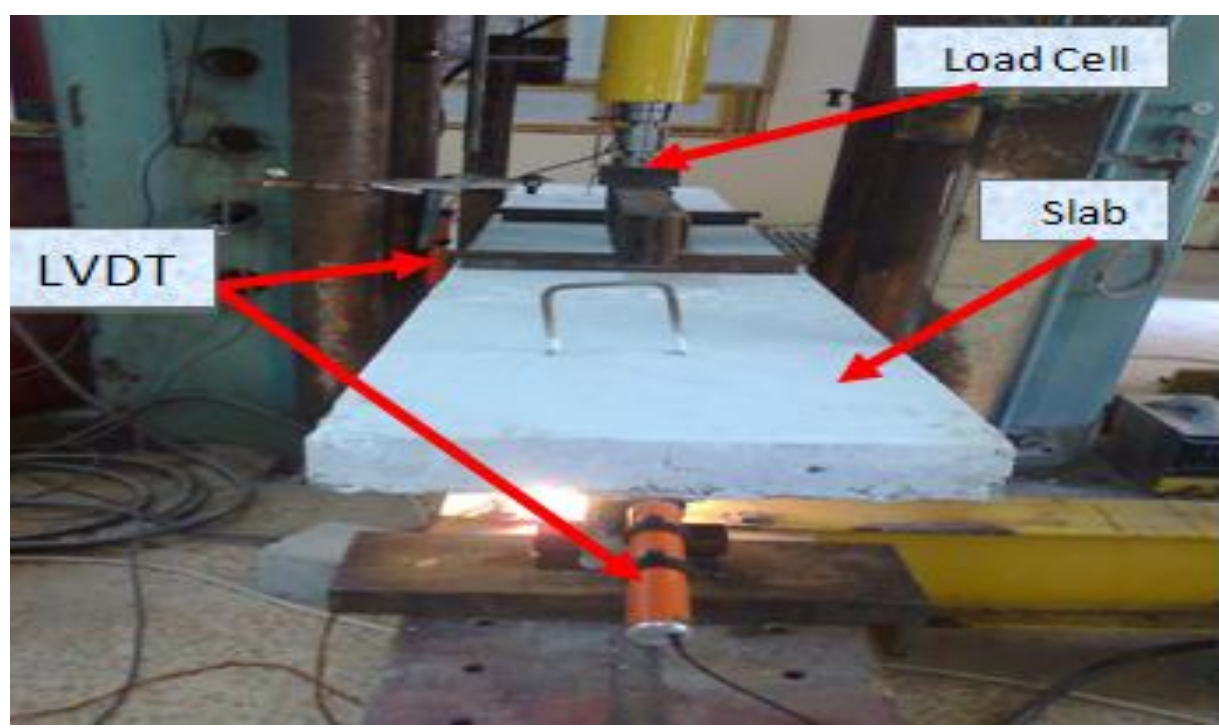

الشكل(4): العتبة المركبة على جهاز الفحص.

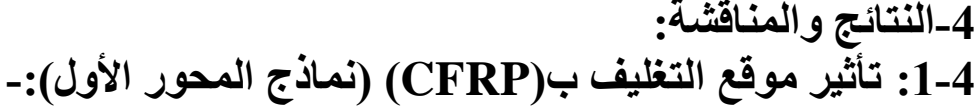

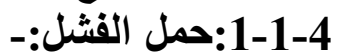

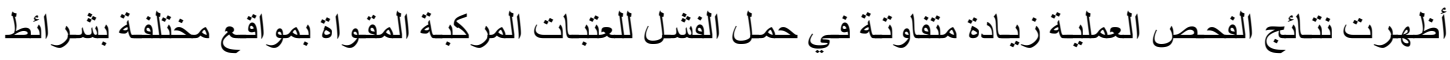

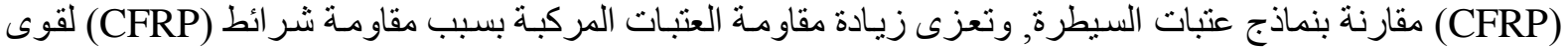

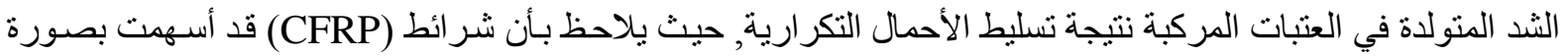

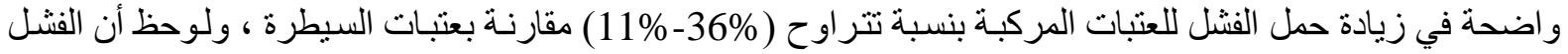

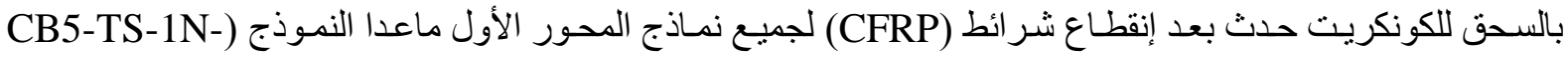

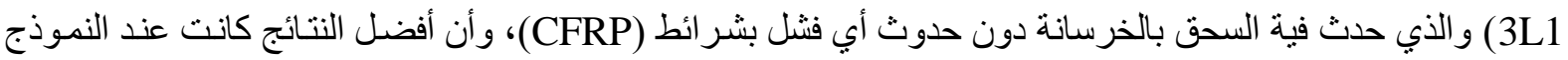

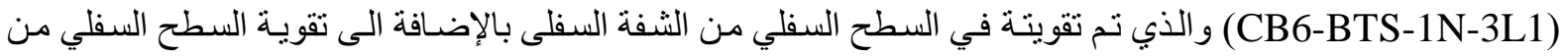

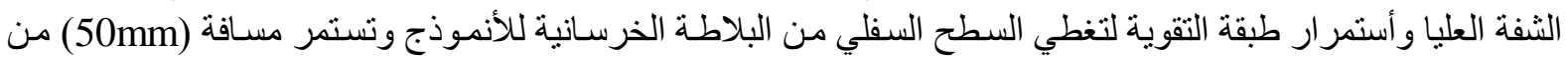

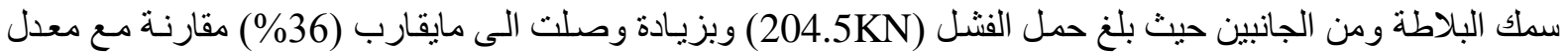

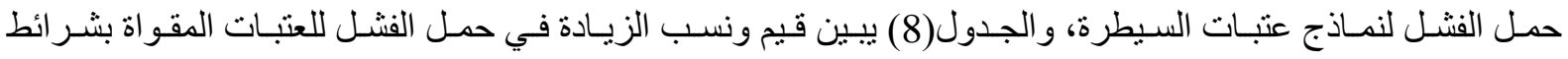
مقارنة مع معدل حمل الفتشل لعتبات السيطرة. 


$$
\text { جدول(8):قيم ونسب الزيادة في حمل الفشل لنماذج المحور الأول. }
$$

\begin{tabular}{|c|c|c|c|}
\hline Composite Beams & $\begin{array}{c}\text { Failure Load } \\
(\mathbf{k N})\end{array}$ & $\begin{array}{c}\text { \% Increasing in Failure } \\
\text { Load With Respect to } \\
\text { Control Beams }\end{array}$ & Mode Of Failure \\
\hline Avg. of CCB2 \& CCB1 & 150.4 & ---- & Crushing In Concrete \\
\hline CB1-B-1N-3L1 & 166.9 & 11 & Rupture In CFRP \\
\hline CB2-B-1N-3L1 & 174.4 & 16 & Rupture In CFRP \\
\hline CB3-BW-1N-3L1 & 175.2 & 17 & Rupture In CFRP \\
\hline CB4-BU-1N-3L1 & 172.7 & 15 & Rupture In CFRP \\
\hline CB5-TS-1N-3L1 & 189.8 & 26 & Crushing In Concrete \\
\hline CB6-BTS-1N-3L1 & 204.5 & 36 & Rupture In CFRP \\
\hline
\end{tabular}

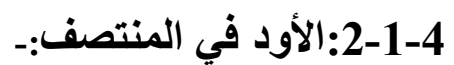

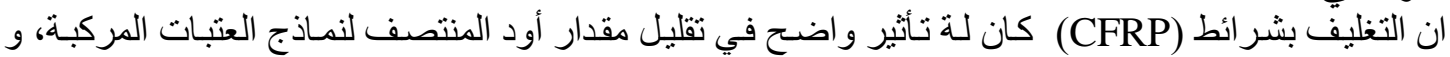

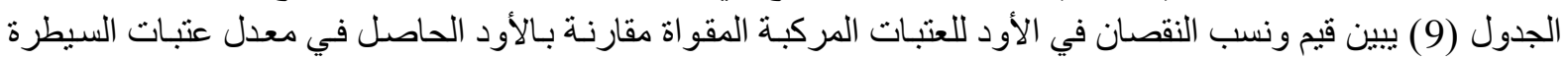

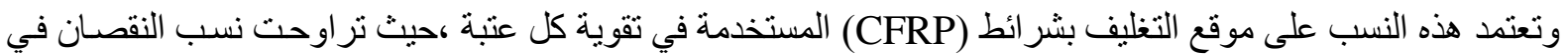

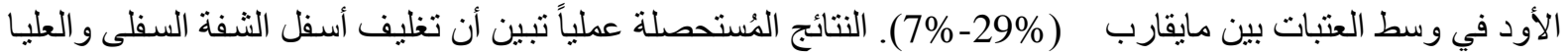

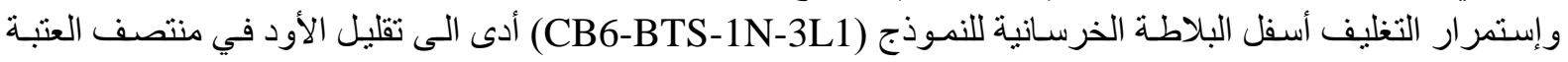

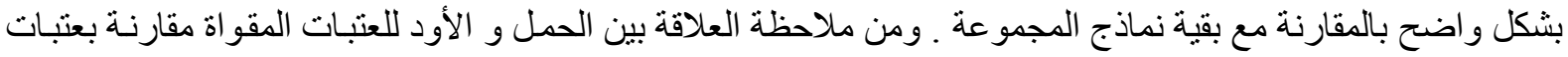

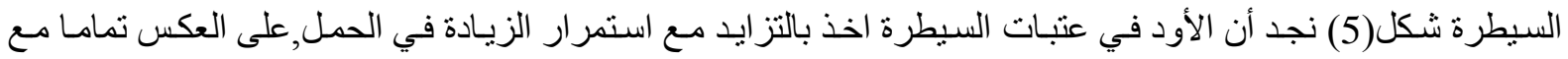

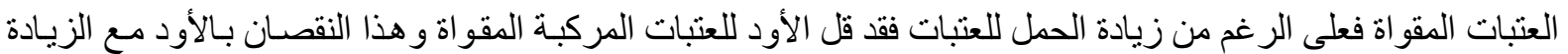

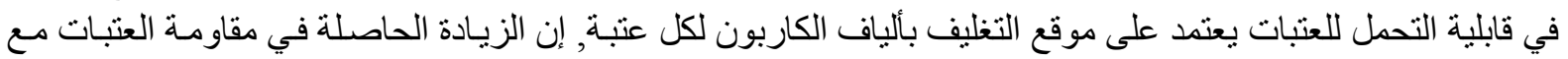
نقصان بالأود الحاصل سبية يعود الى كون شر ائط (CFRP) قد عملت على تحمل قوى الثد في أسفل العتبة و عملت على التى

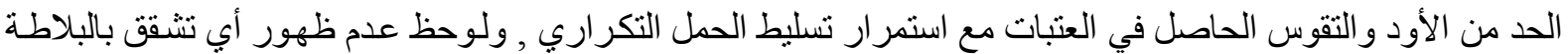

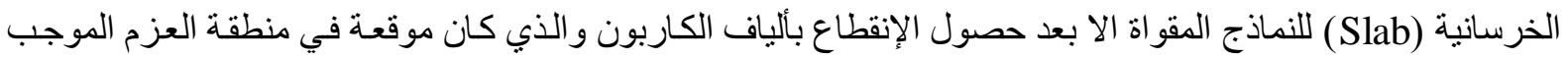

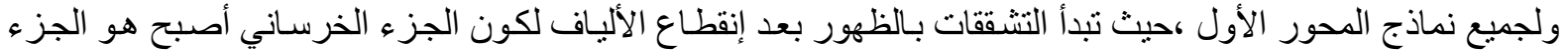

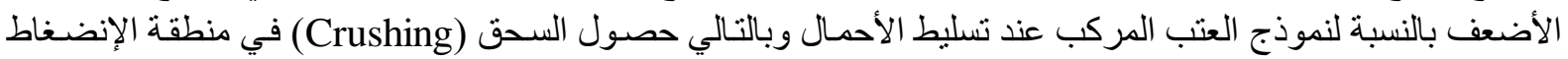
مع حصول تقوس واضح بعد قطع الألياف يحدث في المقطع الحديدي لنماذج العتبات المركبة.

جدول(9): قيم الأود في وسط العتبات مع نسب النقصان للعتبات المقواة ضمن نماذج المحور الأول بالمقارنة مع أعتاب السيطرة.

\begin{tabular}{|c|c|c|}
\hline Composite Beams & $\begin{array}{c}\text { Mid Span Deflection } \\
(\mathbf{m m})\end{array}$ & $\begin{array}{c}\text { \%Decreasing In Mid Span } \\
\text { Deflection } \\
\text { (At Failure Load) }\end{array}$ \\
\hline Avg. of CCB1\&CCB2 & 75.2 & -- \\
\hline CB1-B-1N-3L1 & 70.5 & 7 \\
\hline CB2-B-1N-3L1 & $\mathbf{6 8 . 2}$ & 9 \\
\hline CB3-BW-1N-3L1 & $\mathbf{6 5 . 6}$ & $\mathbf{1 2}$ \\
\hline CB4-BU-1N-3L1 & $\mathbf{6 9 . 3}$ & $\mathbf{8}$ \\
\hline CB5-TS-1N-3L1 & $\mathbf{6 0 . 6}$ & $\mathbf{2 9}$ \\
\hline CB6-BTS-1N-3L1 & $\mathbf{5 3 . 7}$ & \\
\hline
\end{tabular}



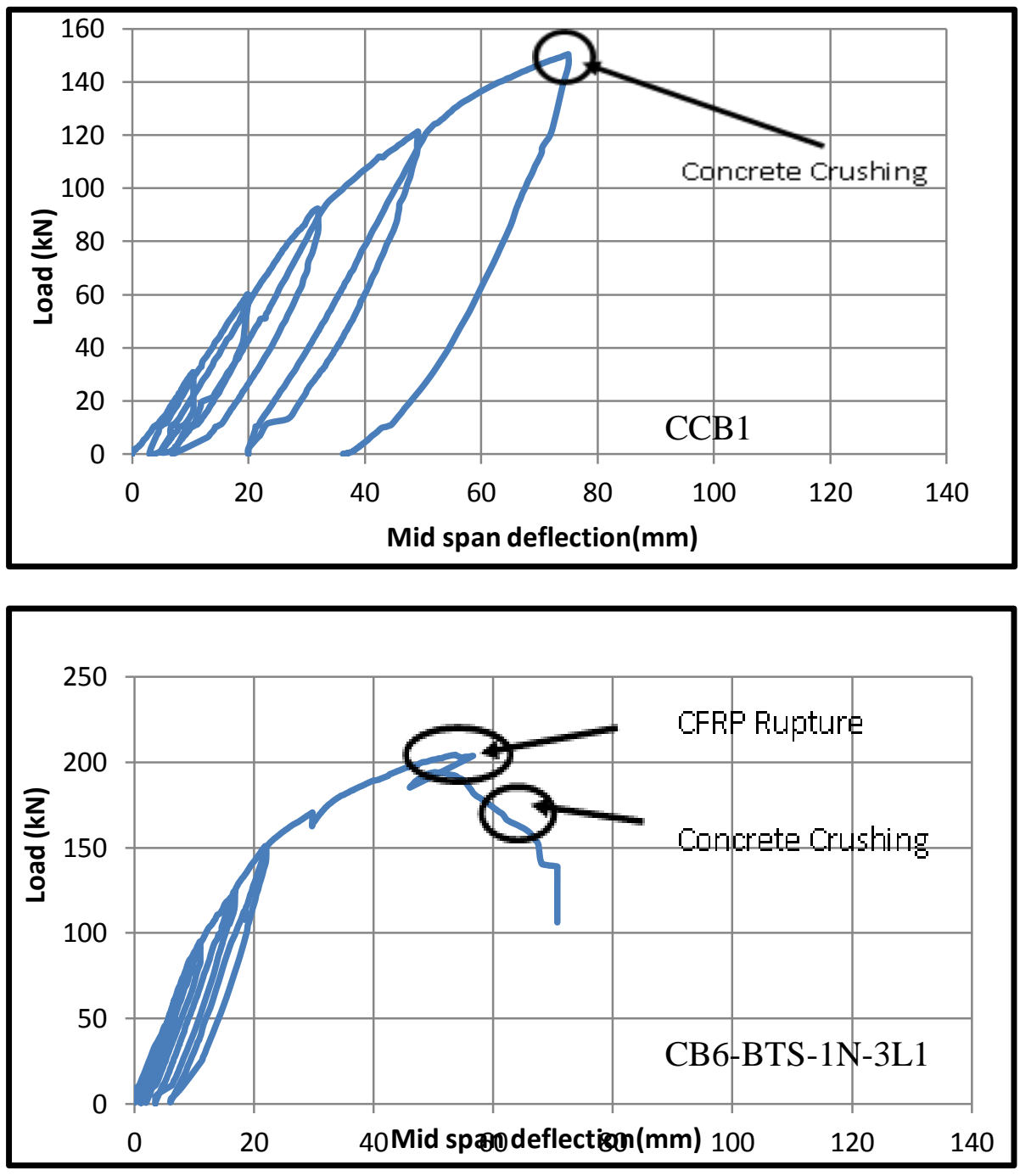

شكل(5): علاقة(الحمل-الأود) للنماذج CCB1\&CB6-BTS-1N-3L1

3-1-4:الإنزلاق عند النهايات:-ولغرض دراسة قوى الربط بين البلاطة الخرسانية و المقطع الحديدي لنماذج العتبات

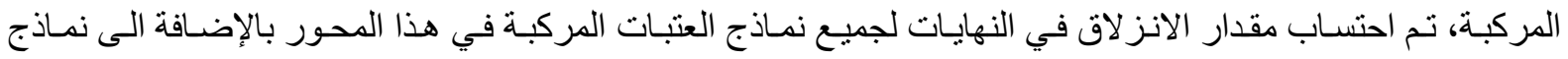

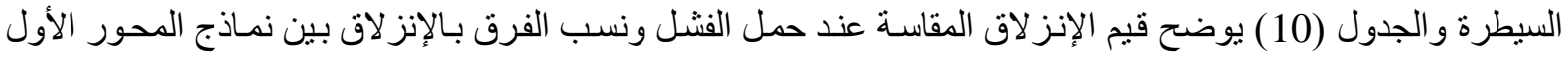

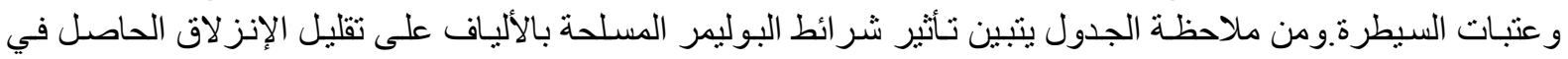

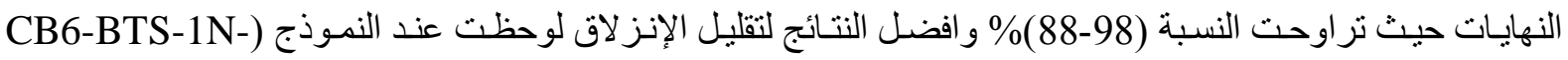

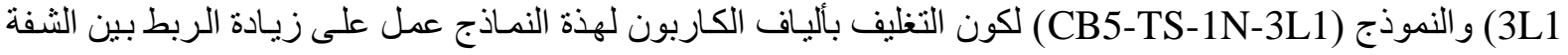

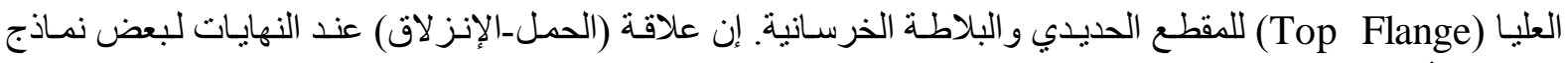

المحور الأول موضحة في الثكل(6). 
ألسليفاني: سلوك العتبات المركبة (حديدخرسانة) المقواة بشرائط (CFRP) تحت تأثير الأحمال التكرارية

جدول(10): قيم الإنزلاق في النهايات ونسب النقصان فيه ضمن نماذج المحور الأول بالمقارنة مع عتبات السيطرة.

\begin{tabular}{|c|c|c|c|}
\hline Composite Beams & Failure Load $(\mathbf{k N})$ & $\begin{array}{c}\text { Slip At Failure } \\
\text { Load(mm) }\end{array}$ & $\begin{array}{c}\text { \%Decreasing in } \\
\text { SlipWith Respect to } \\
\text { Control Beams }\end{array}$ \\
\hline Avg. of CCB1\&CCB2 & 150.4 & $\mathbf{6 . 4 6}$ & ---- \\
\hline CB1-B-1N-3L1 & 166.9 & $\mathbf{0 . 7 6}$ & $\mathbf{8 8}$ \\
\hline CB2-B-1N-3L1 & 174.4 & $\mathbf{0 . 6 4}$ & 90 \\
\hline CB3-BW-1N-3L1 & 175.2 & $\mathbf{0 . 4 5}$ & 93 \\
\hline CB4-BU-1N-3L1 & 172.7 & $\mathbf{0 . 5 8}$ & 91 \\
\hline CB5-TS-1N-3L1 & 189.8 & $\mathbf{0 . 3 2}$ & 95 \\
\hline CB6-BTS-1N-3L1 & $\mathbf{2 0 4 . 5}$ & $\mathbf{0 . 1 3}$ & 98 \\
\hline
\end{tabular}
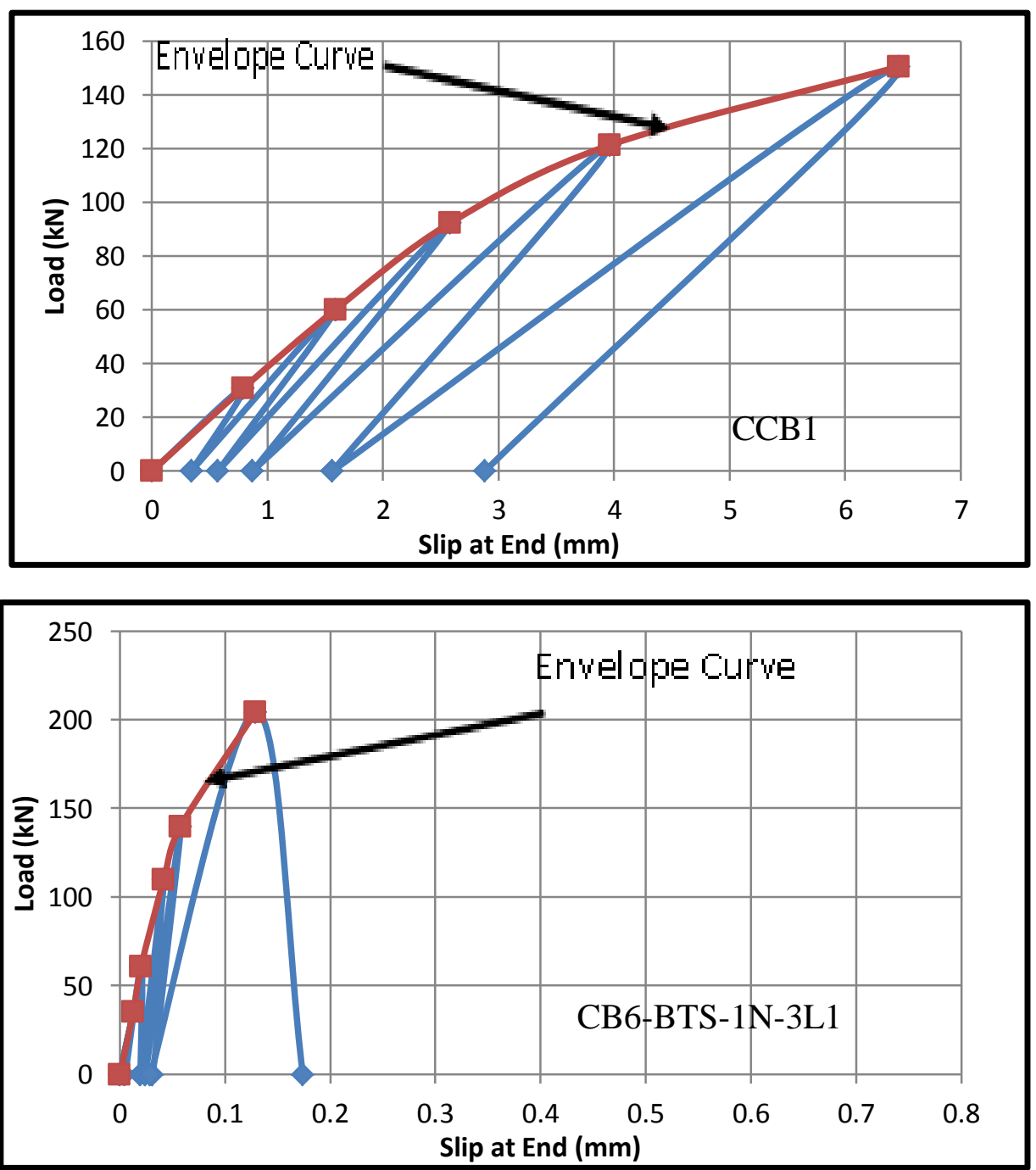

شكل(6): علاقة(الحمل-الإنزلاق)عند النهايات للنماذجCCB1\&CB6-BTS-1N-3L1

2-4:تأثير طول التظليف بشرائط (CFRP) (نماذج المحور الثاني):-

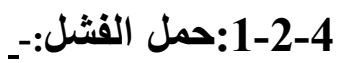

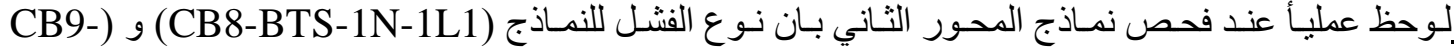

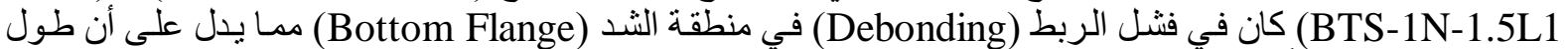

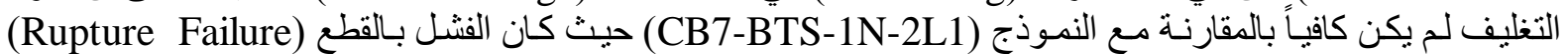

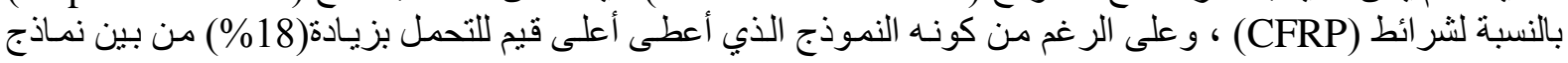


المحور الثاني لكنها نسبة قلبلة بالمقارنـة مع النموذج (CB6-BTS-1N-3L1) و الذي وصلت الزيادة في تحمل الإنثناء

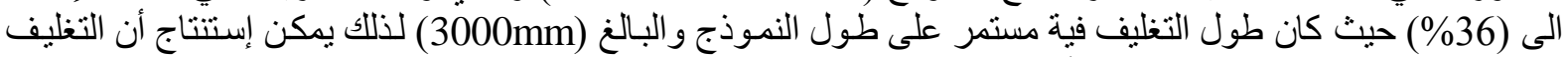

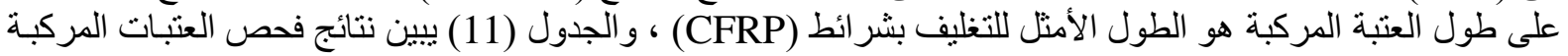

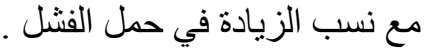

جدول (11) : قيم ونسب الزيادة في حمل الفثل لنماذج المحور الثاني

\begin{tabular}{|c|c|c|c|}
\hline Composite Beams & $\begin{array}{c}\text { Failure Load } \\
(\mathbf{k N})\end{array}$ & $\begin{array}{c}\text { \% Increasing in Failure } \\
\text { Load With Respect to } \\
\text { Control Beams }\end{array}$ & Mode Of Failure \\
\hline Avg. of CCB1\&CCB2 & 150.4 & ---- & Crushing In Concrete \\
\hline CB7-BTS-1N-2L1 & 176.8 & 18 & Rupture In CFRP \\
\hline CB8-BTS-1N-1L1 & 173.2 & 15 & Debonding In CFRP \\
\hline CB9-BTS-1N-1.5L1 & 166.2 & 11 & Debonding In CFRP \\
\hline
\end{tabular}

2-2-4

على الرغم من كون طول المنفول التغليف غير مستمر في نماذج المحور الثاني الا أن ألياف الكاربون عملت على تقليل

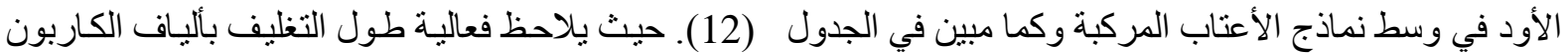

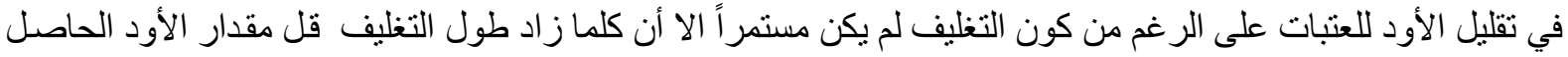

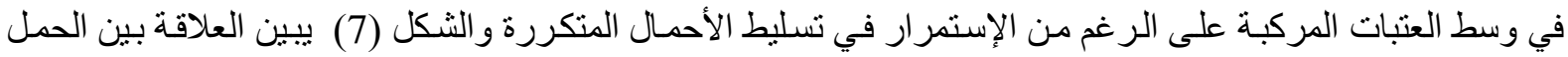

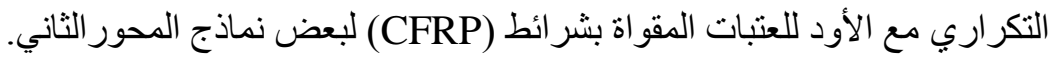
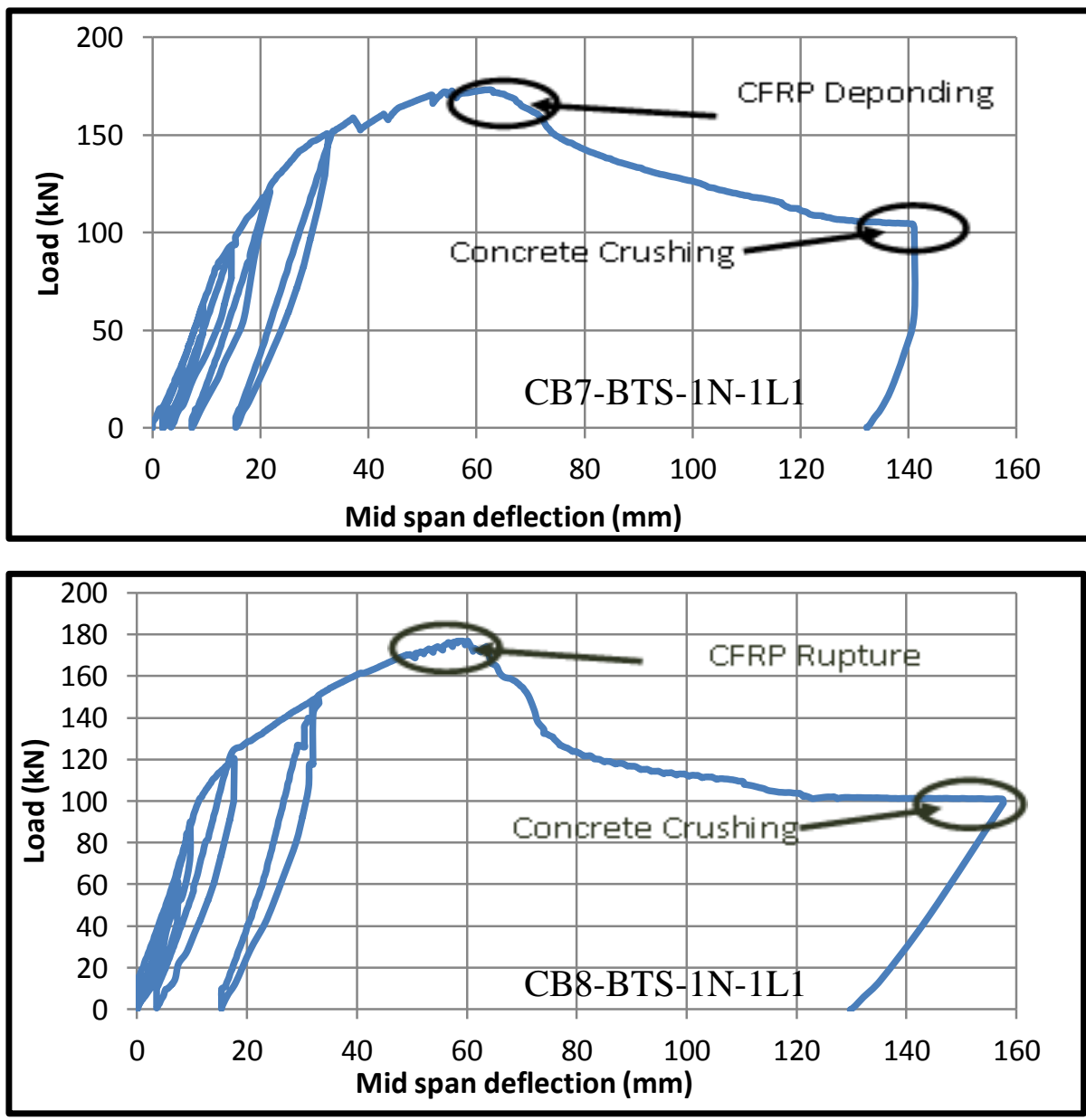

شكل(7): علاقة(الحمل-الأود) للنماذج2B8-BTS-1N-1L1\&CB7-BTS-1N-2L1) 
ألسليفاني: سلوك العتبات المركبة (حديدخرسانة) المقواة بشرائط (CFRP) تحت تأثير الأحمال التكرارية

جدول(12): قيم الأود في وسط العتبات مع نسب النقصان فيه للعتبات المقواة ضمن نماذج المحور

الثانى بالمقارنة مع أعتاب السيطرة..

\begin{tabular}{|c|c|c|}
\hline Composite Beams & $\begin{array}{c}\text { Mid Span Deflection } \\
(\mathbf{m m})\end{array}$ & $\begin{array}{c}\text { \% Decreasing In Mid Span Deflection } \\
\text { (At Failure Load) }\end{array}$ \\
\hline Avg. of CCB1\&CCB2 & 75.2 & - \\
\hline CB7-BTS-1N-2L1 & 60.2 & 20 \\
\hline CB8-BTS-1N-1L1 & 62.5 & 17 \\
\hline CB9-BTS-1N-1.5L1 & 68.5 & 9 \\
\hline
\end{tabular}

3-2-4:الإنزلاق عند النهايات:-

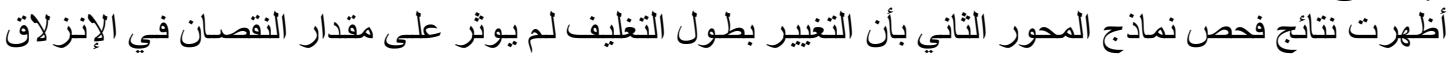

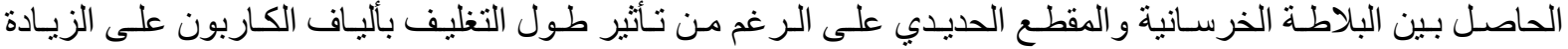

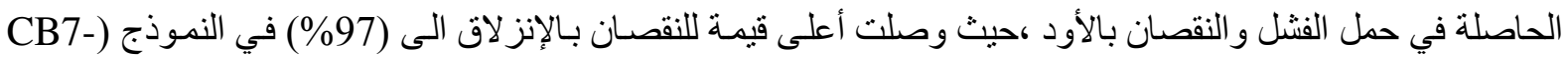
(BTS-1N-2L1

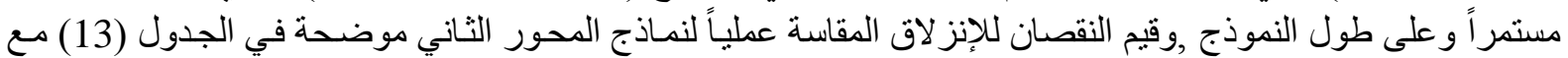

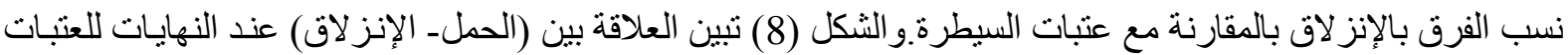

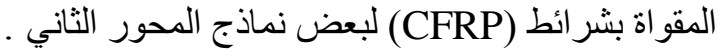
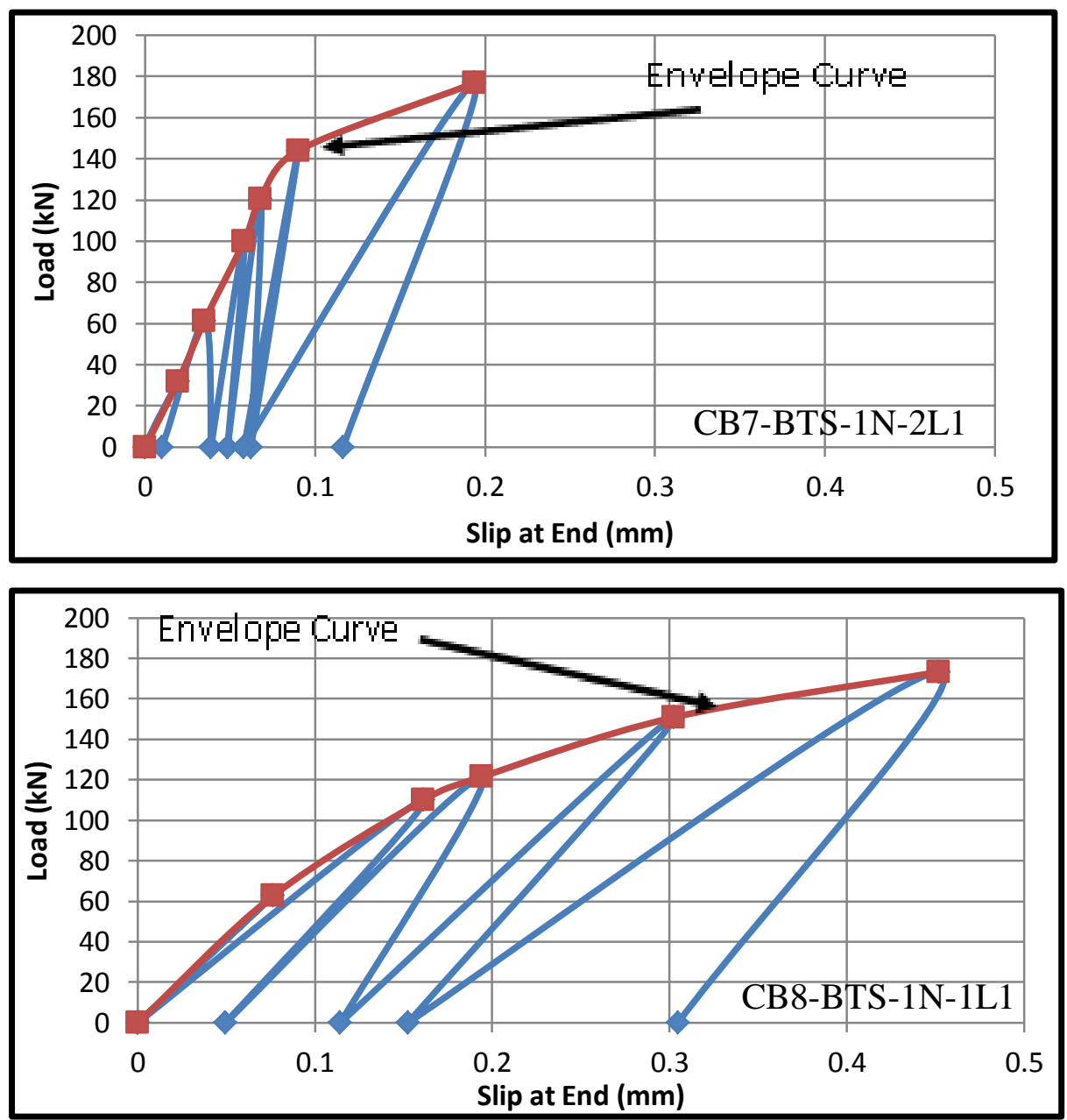

شكل(8): علاقة (الحمل-الإتزلاق)عند النهايات للنماذج-CB8-BTS-1N-1L1\&CB7-BTS 
جدول(13): قيم الإنزلاق في النهايات ونسب النقصان فية ضمن نماذج المحور الثاني بالمقارنة مع عتبات السيطرة.

\begin{tabular}{|c|c|c|c|}
\hline Composite Beams & $\begin{array}{c}\text { Failure Load } \\
(\mathbf{k N})\end{array}$ & $\begin{array}{c}\text { Slip At Failure } \\
\text { Load }(\mathbf{m m})\end{array}$ & $\begin{array}{c}\text { \% Decreasing in SlipWith } \\
\text { Respect to Control Beams }\end{array}$ \\
\hline Avg. of CCB1\&CCB2 & 150.4 & 6.46 & - \\
\hline CB7-BTS-1N-2L1 & 176.8 & 0.19 & 97 \\
\hline CB8-BTS-1N-1L1 & 173.2 & 0.45 & 93 \\
\hline CB9-BTS-1N-1.5L1 & 166.2 & 0.38 & 94 \\
\hline
\end{tabular}

\section{3-4:3-4:ثير عدد طبقات التظليف بشرائط (CFRP) (نماذج المحور الثالث):-}

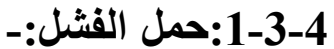

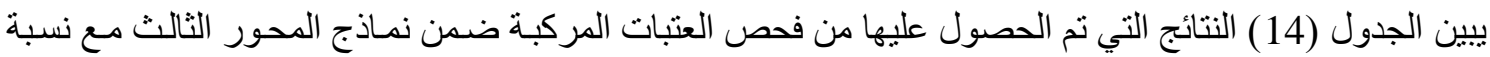

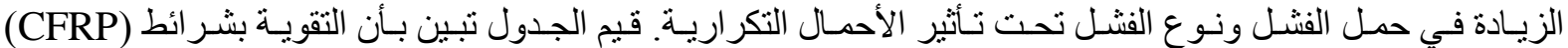
وبطول(3000mm) للنموذج (CB10-BTS-2N-3L1-3L2) أعطت أعلى زيادة في حمل الفشل لنماذج المحور الثالث

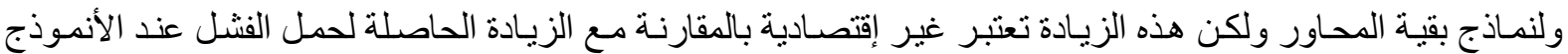
(CB6-BTS-1N-3L1)

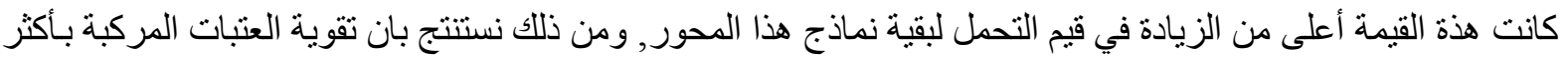
من طبقة لاتؤدي دائما الى زيادة في قابلية التحمل للإنثناء.

جدول(14): قيم ونسب الزيادة في حمل الفشل لنماذج المحور الثالث.

\begin{tabular}{|c|c|c|c|}
\hline Composite Beams & $\begin{array}{c}\text { Failure } \\
\text { Load }(\mathrm{kN})\end{array}$ & $\begin{array}{c}\text { \% Increasing in Failure Load } \\
\text { With Respect to Control Beams }\end{array}$ & Mode Of Failure \\
\hline Avg. of CCB1\&CCB2 & 150.4 & ----- & $\begin{array}{c}\text { Crushing In } \\
\text { Concrete }\end{array}$ \\
\hline CB10-BTS-2N-3L1-3L2 & 206 & 37 & Rupture In CFRP \\
\hline CB11-BTS-2N-3L1-2L2 & 200 & 33 & Rupture In CFRP \\
\hline CB12-BTS-2N-2L1-2L2 & 203 & 35 & Rupture In CFRP \\
\hline
\end{tabular}

\section{2-3-4:الأود في المنتصف:-}

يبين الجذول(15) قيم ونسب النقصان في الأود للعتبات المقواة بطبقتين وبأطو ال مختلفة من شر ائط (CFRP)

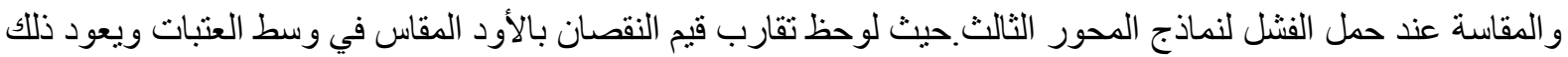

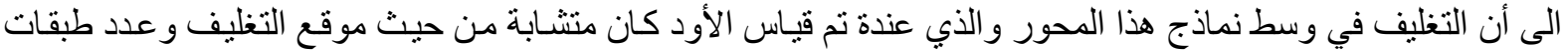

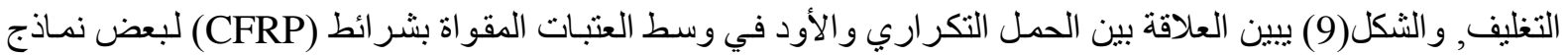

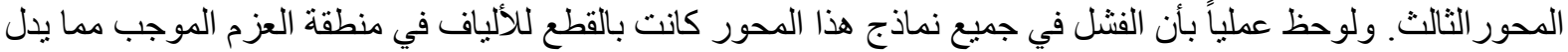

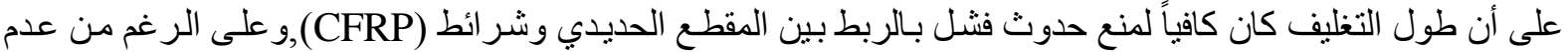

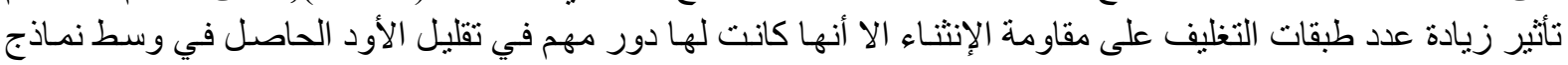
المحور الثالث بالمقارنة مع النقصان الحاصل لنماذج بقية المحاور .

جدول(15): قيم الأود فى وسط العتبات لنماذج المحور الثالث.

\begin{tabular}{|c|c|c|c|}
\hline Composite Beams & $\begin{array}{c}\text { Failure Load } \\
(\mathbf{k N})\end{array}$ & $\begin{array}{c}\text { MidSpan Deflection } \\
(\mathbf{m m})\end{array}$ & $\begin{array}{c}\text { \%Decreasing In Mid Span } \\
\text { Deflection (At Failure Load) }\end{array}$ \\
\hline Avg. of CCB1\&CCB2 & $\mathbf{1 5 0 . 4}$ & $\mathbf{7 5 . 2}$ & $-\mathbf{- 2}$ \\
\hline CB10-BTS-2N-3L1-3L2 & $\mathbf{2 0 6}$ & $\mathbf{3 3 . 1}$ & $\mathbf{5 6}$ \\
\hline CB11-BTS-2N-3L1-2L2 & $\mathbf{2 0 0}$ & $\mathbf{3 3 . 8}$ & $\mathbf{5 5}$ \\
\hline CB12-BTS-2N-2L1-2L2 & $\mathbf{2 0 3}$ & $\mathbf{3 3 . 1}$ & $\mathbf{5 6}$ \\
\hline
\end{tabular}



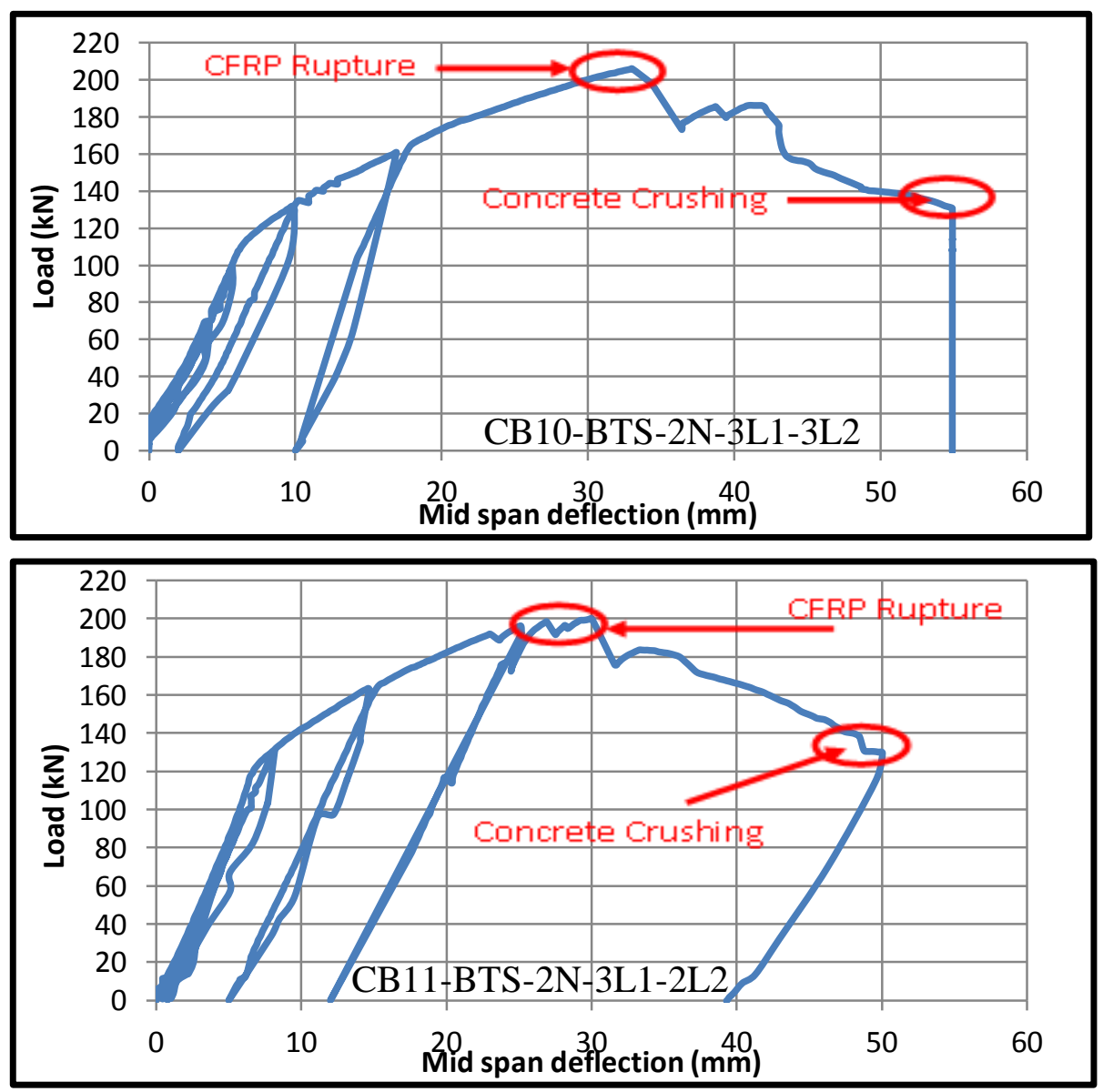

شكل(9): علاقة(الحمل-الأود) للنماذجCB10-BTS-2N-3L1-3L2\&CB11-BTS-2N-3L1-2L2

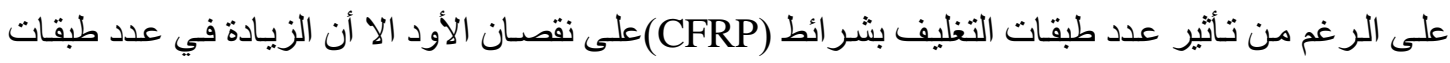

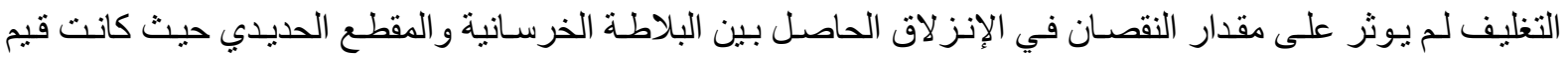

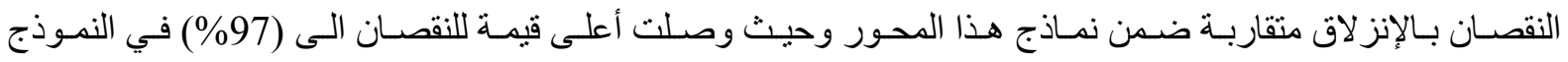

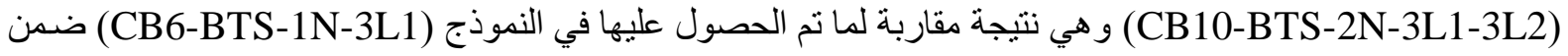

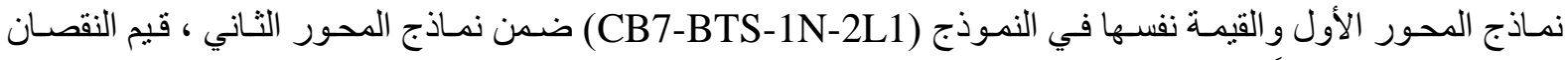

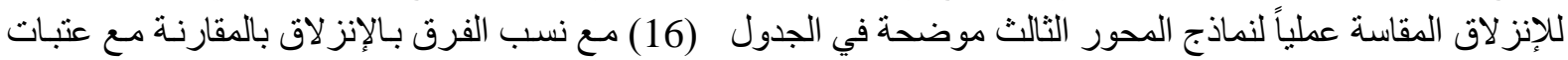

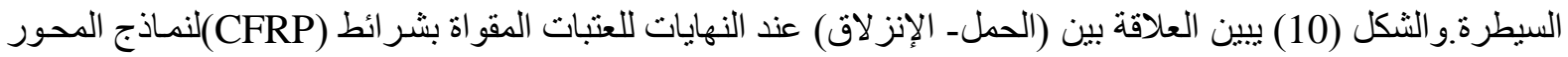

جدول(16): قيم الإنزلاق في نهايات نماذج المحور الثالث

\begin{tabular}{|c|c|c|c|}
\hline Composite Beams & $\begin{array}{c}\text { Failure Load } \\
(\mathbf{k N})\end{array}$ & $\begin{array}{c}\text { Slip at Failure } \\
\text { Load(mm })\end{array}$ & $\begin{array}{c}\text { \% Decreasing in SlipWith Respect to } \\
\text { Control Beams }\end{array}$ \\
\hline Avg. of CCB1\&CCB2 & $\mathbf{1 5 0 . 4}$ & $\mathbf{6 . 4 6}$ & $-\cdots----$ \\
\hline CB10-BTS-2N-3L1-3L2 & 206 & $\mathbf{0 . 1 9}$ & 97 \\
\hline CB11-BTS-2N-3L1-2L2 & 200 & $\mathbf{0 . 3 2}$ & 95 \\
\hline CB12-BTS-2N-2L1-2L2 & 203 & 0.26 & 96 \\
\hline
\end{tabular}



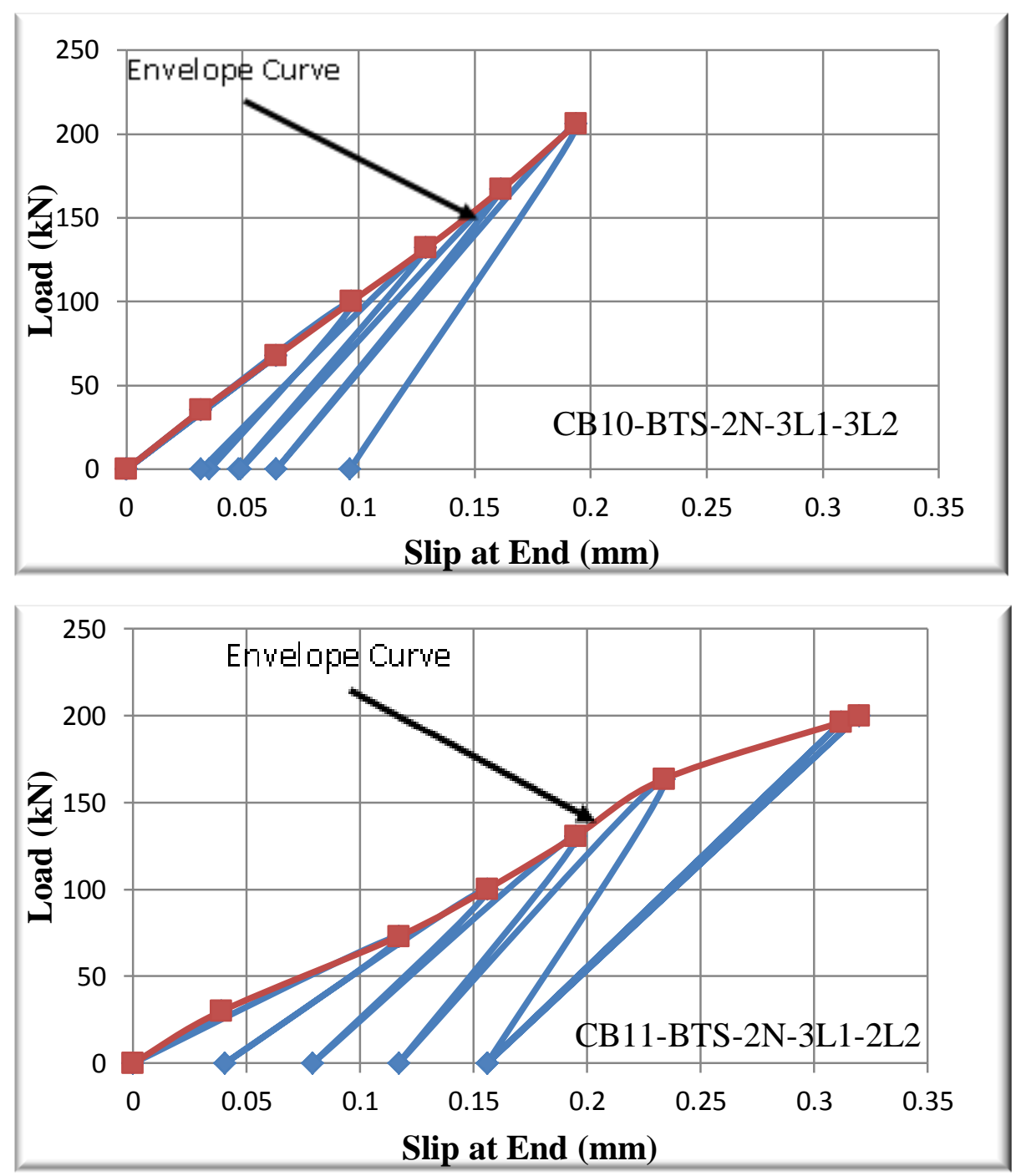

\section{شكل(10): (الحمل-الإنزلاق) للنماذجCB10-BTS-2N-3L1-3L2\&CB11-BTS-2N-3L1-2L2}

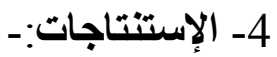

ـ لثر ائط (CFRP) قابلية جيدة على تقوية مقاومة الانثناء للعتبات المركبة، فقد اسهمت في زيادة مقاومة العتبات المركبة

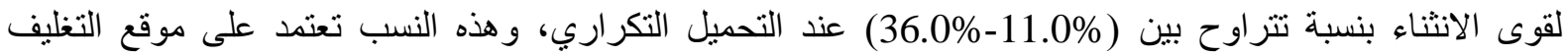
بالألياف.

2- أسهمت شرائط (CFRP) في تقليل الأود المقاس في منتصف العتبات المركبة وبنسب تتراوح بين (56\%-7\%)

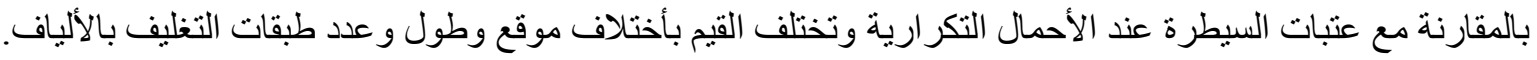
3- التغليف باكثر من طبقة واحدة من شر ائط (CFRP) تعطي زيادة طفيفة ضد مقاومة الانثناء فضلاً عن انها لاتعد مجدية من الناحية الاقتصادية.

4- ان تغليف الأعتاب المركبة بشرائط (CFRP) كان له تأثير واضح بتقليل الإنزلاق الحاصل بين المقطع الحديدي

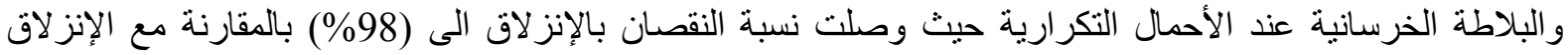
الحاصل في عتبات السيطرة . 


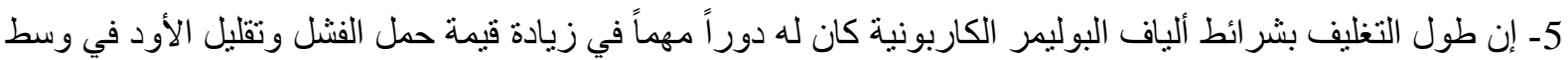

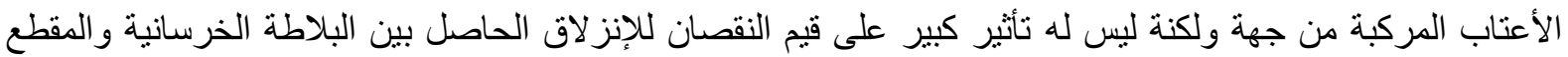

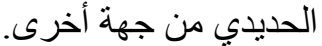
| (المصادر:-

[1] Al-Saffar, I.S., "Nonlinear Analysis for Composite Steel-Concrete Beam Elements under Repeated Loads", M.Sc. Thesis, University of Mosul, Iraq, 2006.

[2] Ragab,N.A.,"Strengtheing of Steel-Concrete Composite Girders Using Various Advanced Composite Matirals" M.Sc.,Thesis, University of Calgargy,Alberta,Canada,2007.

[3] Tavakkolizadeh M.,Saadatmanesh H.,"Strengthening of Steel-Concrete Composite Girder Using Carbon Fiber Reinforced Polymers Sheets",Journal of Structral Engineering,ASCE,Vol. 129,No.1,January 1,2003,pp.30-40.

[4] Spacone,E.,and Ei-Tawil,S., "Nonlinear Analysis of Steel-Concrete Composite Structures : State of', Journal of the Structural Engineering ,Vol.130,No.2,February 2004,pp.159168.

[5] Teng J.G.,Chen J.F.,Smith S.T.,and Lam L.,"FRP Strengthened RC Structures",John Wiley\&Sons,Ltd,England,2002,245pp.

[6] المواصفات القياسية العر اقية (رقم 5)، (1984)، "خصائص الإسمنت البورتلاندي الاعتيادي“، الجهاز المركزي اللتقييس و السيطرة النوعية، بغداد، العراق، 1984.

[7] B.S.882-1992,“Aggregates from Natural Source for Concrete”, British Standard Institution, 1992.

[8] ASTM C39-04,"Compressive Strength of Cylindrical Concrete Specimens",American Society for Testing and Materials, 2004.

[9] ASTM C617-98,(Reapproved 2003),"Capping Cylindrical Concrete Specimens",American Society for Testing and Materials, 2003.

[10] AISC,"Specification for Structural Steel Buildings",ANSI/AISC 360-05,American Institute of Steel Construction, Inc.,One East Wacker Drive,Suite 700,Chicago,Illinois,USA,2005.

[11] Sika Data Book Construction "Building with a Safe System",2005, www.Sikaneareast.com

[12] "Structural Strengthening with Sika Wrap Fabric System", Sika Services AGCH- 8048 Zurich, Switzerland, p.415, www.Sika.com.

[36] Wright,H.D.,Harding P.W.,and Evans H.R.,"The use of Pushout Tests to Simulate Shear Connectors in Composite Beam Construction", University College,Cardiff, UK,pp39-47.

[14] BS 5400, Part 5, "Steel, Concrete and Composite Bridges", British Standards Institution, London, 1979.

تم اجراء البحث في كلية ألهندسة = جامعة ألموصل 